\title{
Mitochondrial Efficiency-Dependent Viability of Saccharomyces cerevisiae Mutants Carrying Individual Electron Transport Chain Component Deletions
}

\author{
Young-Yon Kwon', Kyung-Mi Choi', ChangYeon Cho', and Cheol-Koo Lee ${ }^{1, *}$
}

\begin{abstract}
Mitochondria play a crucial role in eukaryotic cells; the mitochondrial electron transport chain (ETC) generates adenosine triphosphate (ATP), which serves as an energy source for numerous critical cellular activities. However, the ETC also generates deleterious reactive oxygen species (ROS) as a natural byproduct of oxidative phosphorylation. ROS are considered the major cause of aging because they damage proteins, lipids, and DNA by oxidation. We analyzed the chronological life span, growth phenotype, mitochondrial membrane potential (MMP), and intracellular ATP and mitochondrial superoxide levels of 33 single ETC component-deleted strains during the chronological aging process. Among the ETC mutant strains, 14 (sdh1 $\Delta$, sdh2 $\Delta$, sdh4 $\Delta$, cor1 $\Delta$, cyt1 qcr7 $\Delta, q c r 8 \Delta$, rip1 $\Delta, \operatorname{cox} 6 \Delta, \operatorname{cox} 7 \Delta, \operatorname{cox} 9 \Delta$, atp $4 \Delta$, atp $7 \Delta$, and atp17 1 ) showed a significantly shorter life span. The deleted genes encode important elements of the ETC components succinate dehydrogenase (complex II) and cytochrome c oxidase (complex IV), and some of the deletions lead to structural instability of the membrane- $F_{1} F_{0}$ ATP synthase due to mutations in the stator stalk (complex V). These short-lived strains generated higher superoxide levels and produced lower ATP levels without alteration of MMP. In summary, ETC mutations decreased the life span of yeast due to impaired mitochondrial efficiency.
\end{abstract}

\section{INTRODUCTION}

The mitochondrial theory of aging remains to be one of the most popular ones (Passos et al., 2006). In the 1950s, the push

\footnotetext{
${ }^{1}$ Department of Biotechnology, College of Life Sciences and Biotechnology, Korea University, Seoul 136-701, Korea, ${ }^{2}$ Animal Genetic Resources Research Center, National Institute of Animal Science, Rural Development Administration, Jeonbuk 590-832, Korea

*Correspondence: cklee2005@korea.ac.kr
}

Received 2 June, 2015; revised 29 August, 2015; accepted 3 September, 2015; published online 24 November, 2015

Keywords: cellular ATP, electron transport chain, ETC-component single gene deletion, mitochondria, mitochondrial ROS in radiation biology research led to the discovery of oxygen toxicity, and Gerschman showed that the oxygen poisoning observed upon X-ray irradiation produced its lethal effect by forming free radicals in the exposed cells (Gerschman et al., 1954). Harman observed that irradiation generated oxygen radicals that probably caused premature aging. However, normal living cells also produce oxygen radicals. Hence, Harman theorized that endogenously generated oxygen radicals might initiate the aging process (Harman, 1956). In 1961, the respiratory system in mitochondria was first identified and studied at the molecular level (Mitchell, 1961). Fridovich et al. purified superoxide dismutase from bovine erythrocytes, which directly detoxified the superoxide radicals generated as byproducts of the electron transport chain (ETC) (McCord and Fridovich, 1969). Based on these discoveries, Harman proposed the mitochondrion to be the biologic clock in mammals, and these ideas were further developed into a theory called the "mitochondrial theory of aging" (Harman, 1972).

In eukaryotic cells, mitochondria are essential organelles that are the sites of energy production. The primary function of mitochondria is releasing the energy contained in nicotinamide adenine dinucleotide (NADH) through oxidative phosphorylation (Bratic and Larsson, 2013). Mitochondrial function declines with age, which is marked by a decrease in mitochondrial capacity, biogenesis, efficiency, and DNA copy number (Bratic and Larsson, 2013; Choi and Lee, 2013; Choi et al., 2013a; 2013b; 2015). The budding yeast Saccharomyces cerevisiae has been used to discover longevity genes and reveal aging mechanisms (Kaeberlein et al., 2005). Interestingly, S. cerevisiae can live without mitochondria; therefore, it is a good model for studying the mitochondrial respiratory system during aging (Breitenbach et al., 2012). S. cerevisiae is a facultative anaerobe that prefers fermentation in glucose-rich culture media but switches to respiration (diauxic shift) upon glucose depletion. However, mitochondrial respiratory system-deficient mutants are viable and can be easily isolated (Breitenbach Ln et al., 2012). Because $S$. cerevisiae can survive by using fermentation instead of respiration, mutations in genes associated with mitochondrial function in $S$. cerevisiae may have few effects. In a previous microarray study, we identified several genes encoding components of the mitochondrial ETC that were positively correlated with caloric restriction (CR)-associated longevity in S. cerevisiae (Lee and Lee, 2008). Furthermore, 
A

c
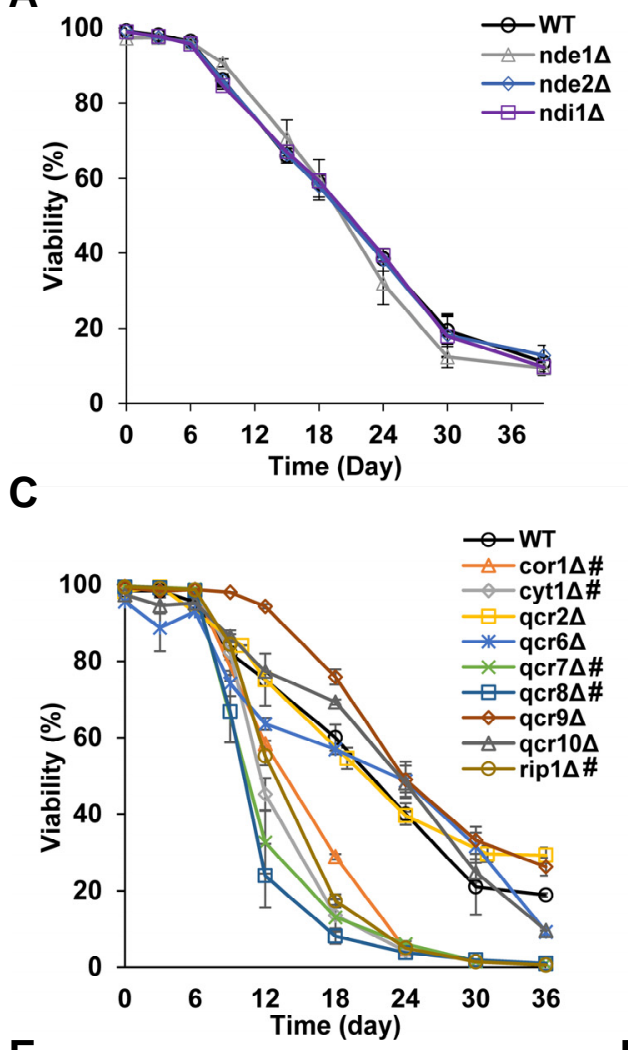

E

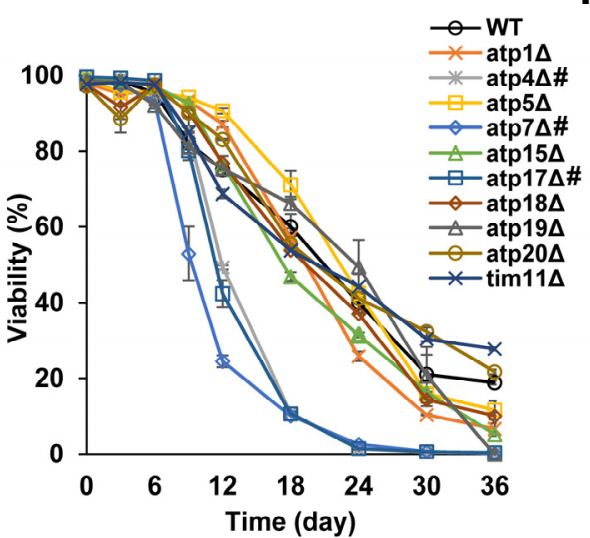

B

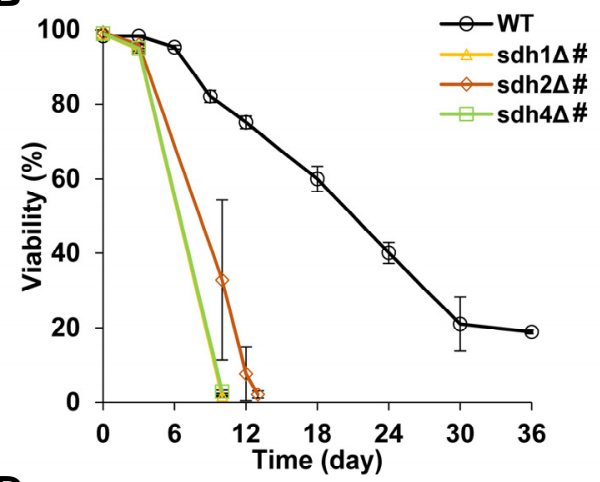

D

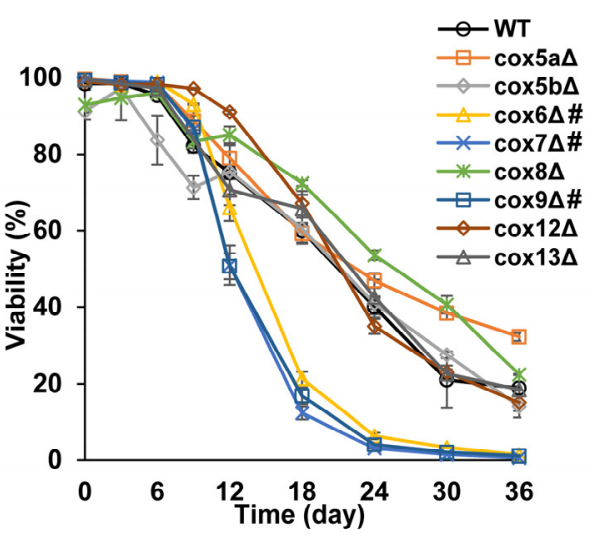

$\boldsymbol{F}$

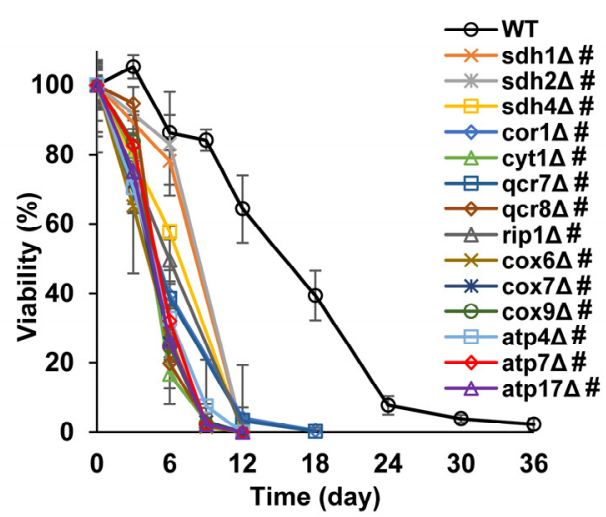

Fig. 1. Viability of S. cerevisiae strains with single deletions in electron transport chain (ETC) genes. (A-E) The chronological lifes pan (CLS) of strains with a single gene deletion in NADH dehydrogenase $(A)$, complex II (B), complex III (C), complex IV (D), and complex $V(E)$ was measured by a propidium iodide $(\mathrm{PI})$ assay. $(\mathrm{F})$ Independent validation by colony forming unit (CFU) assay for 14 short-lived strains selected by abovementioned method. Error bars indicate standard deviation. "Short-lived strains. we confirmed that the protein synthesis of mitochondrial ETC components increased under CR (Choi et al., 2011). Based on these results, we hypothesized that mitochondrial efficiency and capacity might be important factors for life span extension by CR.

The ETC is the workhorse in mitochondria that produces adenosine triphosphate (ATP) through oxidative phosphorylation. However, mitochondria also generate several reactive oxygen species (ROS) as byproducts during this process. According to the mitochondrial free radical theory, aging is caused by oxidative stress resulting from the accumulation of mitochondrial ROS at the cellular and tissue levels (Bratic and Larsson, 2013). The primary mitochondrial ROS is the superoxide radical produced by complexes I and III (Muller et al., 2004). Normally, mitochondrial manganese superoxide dismutase (Mn-SOD) immediately breaks down superoxide radicals into less-reactive hydrogen peroxides (Gralla and Kosman, 1992). The reactivity of superoxide and hydrogen peroxide is not sufficient to directly damage mtDNA (Stehr-Green et al., 1990). However, superoxide can directly damage Fe-S clusters in the ETC components of the mitochondrial inner membrane, mainly complex I, II, and III, even before the antioxidant intervention. Damaged Fe-S clusters release $\mathrm{Fe}^{2+}$ ions, which produce hydroxyl radicals by reacting with mitochondrial superoxides and hydrogen peroxides (Veatch et al., 2009). These hydroxyl radicals can directly damage proteins, fatty acids, and mtDNA (Scialo et al., 2013). Increased damage could cause mitochondrial malfunction, further increasing the generation of ROS (de Grey, 2005). Mitochondrial superoxide levels are strongly correlated with life span, because among all the antioxidant-deficient mutants examined, the Mn-SODdeficient mutant had the shortest life span during chronological 

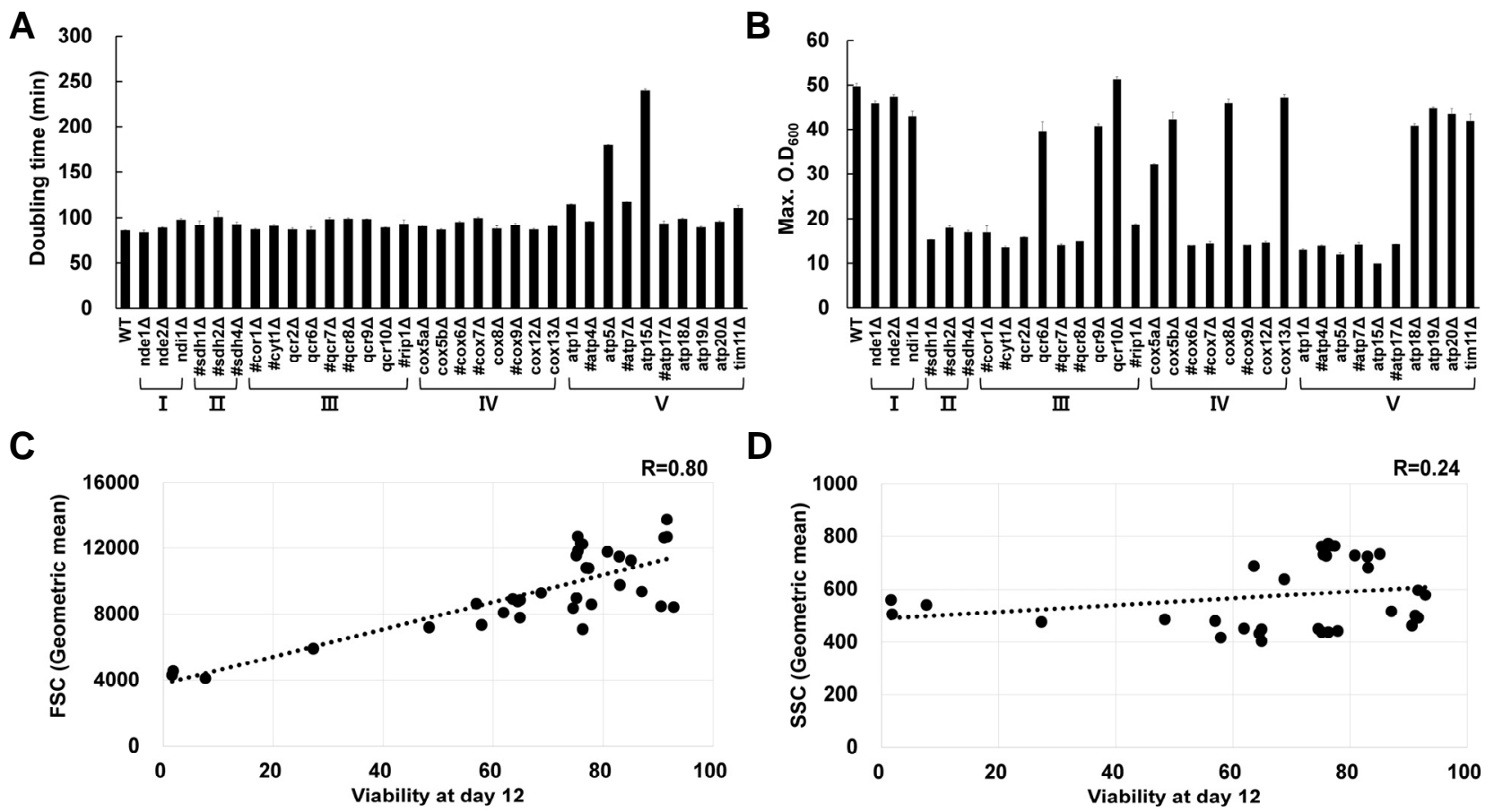

$D$

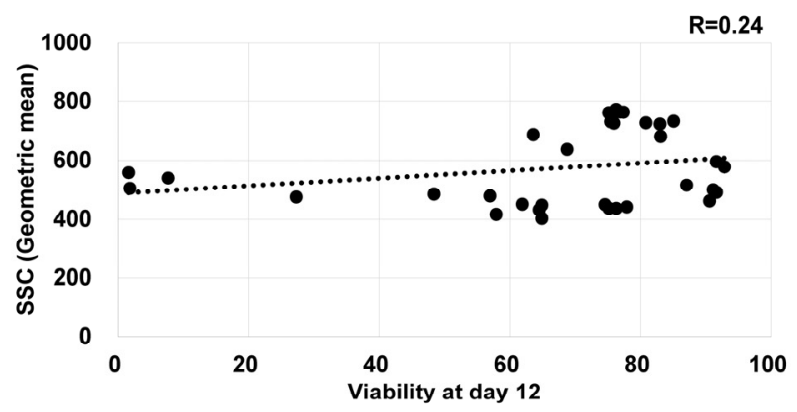

Fig. 2. Growth phenotype of mutants with ETC single component deletions. (A, B)Cell doubling time during exponential growth (A) and maximum cell mass (B) of ETC single gene deletion mutants. (C) Correlation between viability and cell size. Forward-scattered light (FSC) of flow cytometry was used for cell size. (D) Correlation between viability and cell complexity. Side-scattered light (SSC) of flow cytometry was used for cell complexity. Error bars indicate standard deviation. "Short-lived strains.

aging (Demir and Koc, 2010). However, the effect of the MnSOD mutation was not consistent in all species. Although MnSOD mutations decrease the life span of flies (Duttaroy et al., 2003) and mice (Li et al., 1995), much like that of budding yeast, this mutation extended the life span of worms (Van Raamsdonk and Hekimi, 2009).

Here, we hypothesized that mitochondrial ETC components play important roles in determining the life span of $S$. cerevisiae during chronological aging. Hacioglu et al. measured the replicative life span (RLS) of respiratory chain single gene knock-out (KO) strains (Hacioglu et al., 2012). They found that single gene deletions of NDE1, TCM62, RIP1, CYT1, QCR8, PET117, COX11, ATP11, and FMC1 shortened the RLS compared to that of wild type. Despite measuring ROS production, mitochondrial membrane potential (MMP), and respiration capacity in these short-lived strains, they could not determine the mechanism underlying the shorter RLS (Hacioglu et al., 2012) because the ROS and MMP measurements were obtained at a single point in very early exponential growth phase when the $\mathrm{KO}$ and wild-type strains were indistinguishable. In this study, we systematically analyzed the role of the mitochondrial ETC system during the entire chronological aging process by using the yeast ETC deletion library. The results of our study provide new evidence for the importance of mitochondrial efficiency and respiration in the aging process.

\section{MATERIALS AND METHODS}

\section{Yeast strains and culture conditions}

All S. cerevisiae strains were obtained from the Open Biosystems Yeast library. The strains used include wild type BY4741
(MATa his3 $\Delta 1$ leu2 $\Delta 0$ met15 $\triangle 0$, ura3 $\Delta 0$ ) and 33 isogenic single deletion mutants (nde1 $\Delta$, nde $2 \Delta$, ndi1 $\Delta$, sdh1 $\Delta$, sdh2 $\Delta$, sdh4 $\Delta$,

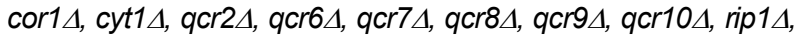
$\operatorname{cox} 5 a \Delta, \operatorname{cox} 5 b \Delta, \operatorname{cox} 6 \Delta, \operatorname{cox} 7 \Delta, \operatorname{cox} 8 \Delta, \operatorname{cox} 9 \Delta, \operatorname{cox} 12 \Delta, \operatorname{cox} 13 \Delta$ atp1 $\Delta$, atp4 $\Delta$, atp5 $\Delta$, atp7 $\Delta$, atp15 , atp17 , atp18 , atp19 $\Delta$, atp204, and tim114). All strains were validated by polymerase chain reaction-based genotyping with specific primer pairs (Supplementary Fig. S1).

Frozen yeast strains were streaked onto yeast extractpeptone-dextrose (YPD) agar plates containing 2\% Bacto ${ }^{\mathrm{TM}}$ agar, $1 \%$ Bacto $^{\mathrm{TM}}$ yeast extract, $2 \%$ Bacto $^{\mathrm{TM}}$ peptone, and $2 \%$ DifCo $^{\mathrm{TM}}$ dextrose (BD Bioscience, USA), and incubated at $30^{\circ} \mathrm{C}$ to obtain single colonies. Isolated colonies were separately inoculated into $10 \mathrm{ml}$ of YPD medium containing $1 \%$ Bacto $^{\mathrm{TM}}$ yeast extract, $2 \%$ Bacto $^{\mathrm{TM}}$ peptone, and 2\% Difco ${ }^{\mathrm{TM}}$ dextrose and cultured overnight. The seed cultures were inoculated into $10 \mathrm{ml}$ of fresh $2 \%$ glucose-containing YPD medium for $10 \mathrm{~min}$ and subsequently inoculated into $20 \mathrm{ml}$ of medium with the same composition at an initial optical density at $600 \mathrm{~nm}\left(\mathrm{OD}_{600}\right)$ of 0.05 . All yeast cultures were incubated at $30^{\circ} \mathrm{C}$ in an orbital shaker at $200 \mathrm{rpm}$.

\section{Screening for respiratory-deficient strains}

To screen for respiratory deficiency, the 33 mutant strains were spotted on $2 \%$ glucose-containing YPD and 3\% glycerol-containing YPG plates. Overnight cultures were serially diluted to an $\mathrm{OD}_{600}$ of $0.2,0.02,0.002$, and 0.0002 , spotted on YPD and YPG agar plates, and incubated at $30^{\circ} \mathrm{C}$ until colony formation. The colony-bearing plates were imaged using an ImageQuant ${ }^{\mathrm{TM}}$ Las 4000 mini Bio-molecular imager (GE Healthcare, Sweden). 
Table 1. Mean and maximum CLS of S.cerevisiae strains with single deletions in electron transport chain (ETC) genes.

\begin{tabular}{|c|c|c|c|c|c|c|c|c|}
\hline \multirow{2}{*}{ Strain } & \multicolumn{2}{|c|}{ CLS } & \multirow{2}{*}{ Strain } & \multicolumn{2}{|c|}{ CLS } & \multirow{2}{*}{ Strain } & \multicolumn{2}{|c|}{ CLS } \\
\hline & Mean & Max & & Mean & Max & & Mean & Max \\
\hline WT & $21.0 \pm 0.9$ & $>36$ & qcr84 & $10.2 \pm 0.6$ & $17.0 \pm 1.4$ & atp14 & $19.4 \pm 0.4$ & $30.8 \pm 0.5$ \\
\hline nde1A & $20.1 \pm 1.2$ & $>36$ & qcr9s & $23.9 \pm 1.1$ & $>36$ & atp4 4 & $12.0 \pm 0.0$ & $18.6 \pm 0.2$ \\
\hline$n d e 2 \Delta$ & $20.4 \pm 0.8$ & $>36$ & qcr10 $\Delta$ & $23.5 \pm 0.6$ & $35.8 \pm 0.0$ & atp5 & $22.4 \pm 1.0$ & $>36$ \\
\hline ndi1 & $20.8 \pm 0.4$ & $>36$ & rip1s & $12.8 \pm 0.4$ & $21.5 \pm 0.6$ & atp7 4 & $9.3 \pm 0.6$ & $18.1 \pm 0.5$ \\
\hline sdh1A & $6.4 \pm 0.0$ & $9.4 \pm 0.0$ & $\operatorname{cox} 5 a \Delta$ & $22.5 \pm 1.0$ & $>36$ & atp15 & $17.4 \pm 0.2$ & $33.5 \pm 0.2$ \\
\hline$s d h 2 \Delta$ & $8.4 \pm 1.8$ & $11.6 \pm 0.9$ & $\operatorname{cox} 5 b \Delta$ & $21.2 \pm 0.1$ & $>36$ & atp17A & $11.4 \pm 0.3$ & $18.1 \pm 0.1$ \\
\hline$s d h 4 \Delta$ & $6.4 \pm 0.0$ & $9.5 \pm 0.0$ & $\operatorname{cox} 6 \Delta$ & $14.1 \pm 0.4$ & $22.5 \pm 0.4$ & atp184 & $19.2 \pm 0.2$ & $36.1 \pm 1.2$ \\
\hline cor1A & $13.7 \pm 0.1$ & $22.7 \pm 0.2$ & $\operatorname{cox} 7 \Delta$ & $12.2 \pm 0.6$ & $19.4 \pm 1.0$ & atp19A & $24.9 \pm 3.6$ & $>30$ \\
\hline cyt14 & $11.6 \pm 0.4$ & $19.8 \pm 1.7$ & $\operatorname{cox} 8 \Delta$ & $25.7 \pm 0.7$ & $>36$ & atp20 $\Delta$ & $20.3 \pm 0.5$ & $>36$ \\
\hline$q c r 2 \Delta$ & $20.5 \pm 0.6$ & $>36$ & $\operatorname{cox} 9 \Delta$ & $12.2 \pm 0.5$ & $21.1 \pm 0.7$ & $\operatorname{tim} 11 \Delta$ & $20.4 \pm 0.0$ & $>36$ \\
\hline qcr6s & $22.7 \pm 2.4$ & $35.8 \pm 0.3$ & $\operatorname{cox} 12 \Delta$ & $21.2 \pm 0.5$ & $>36$ & & & \\
\hline qcr7 & $10.5 \pm 0.5$ & $20.2 \pm 2.2$ & $\operatorname{cox} 13 \Delta$ & $22.1 \pm 1.1$ & $>36$ & & & \\
\hline
\end{tabular}

Calculation of mean and maximum CLS (10\% surviving) was based on Figs. 1A-1E.

\section{Oxygen consumption rate (OCR)}

OCR was assessed using a Clark-type electrode in samples prepared from cultures on day 1 . Briefly, cells were collected by centrifugation, and the cell pellet was resuspended in $1 \mathrm{ml}$ of $2 \%$ glucose-containing YPD medium. Then, oxygen consumption was measured in these cells using an Oxygraph Plus system (Hansatech instruments, UK) at $30^{\circ} \mathrm{C}$.

\section{Assessment of chronological life span (CLS), and} measurement of MMP and mitochondrial superoxide CLS was assessed using propidium iodide (PI), and MMP and mitochondrial superoxide levels were measured by flowcytometry using 3,3-dihexyloxacarbocyanine iodide (DiOC 6 ) fluorescent dye (Invitrogen, USA) and MitoSOX Red ${ }^{\mathrm{TM}}$ (Invitrogen), respectively, as previously described (Choi et al., 2013b).

\section{Colony-forming unit (CFU) assay}

CFU assay was performed to determine viability, with independent biological repeats for each mutant sample. Cells were harvested and serially diluted in distilled water for cell counting. Diluted cell suspensions were counted on a hemocytometer, and 200 cells were inoculated onto YPD ( $2 \%$ glucose) agar plate. The plates were incubated at $30^{\circ} \mathrm{C}$ until colony formation.

\section{Measurement of intracellular ATP levels}

ATP was extracted from yeast cells by using a previously described method (Choi et al., 2013b). Cultured yeast cells were washed with distilled water 3 times and immediately frozen using liquid $\mathrm{N}_{2}$. Frozen samples were stored at $-70^{\circ} \mathrm{C}$ until use. We measured ATP levels in the extracts according to the manufacturer's protocol (ATP colorimetric/fluorometric kit; Biovision, USA). The excitation and emission wavelengths were $535 \mathrm{~nm}$ and $587 \mathrm{~nm}$, respectively, and fluorescence was measured using a multilabel plate reader (Victor3, Perkin Elmer, USA).

\section{RESULTS}

Effect of ETC component single gene deletions on CLS We determined the CLS of 33 single ETC gene KO mutants (Supplementary Table S1) by using a PI staining method. Of these 33 mutant strains, 14 showed a decreased CLS, whe- reas the CLS of the other KO strains was comparable to that of wild type. Single deletion mutants of the 3 genes encoding NADH dehydrogenase components (nde1 $\Delta$, nde $2 \Delta$, and ndi1 $\Delta$ ) did not alter the CLS. In contrast, the deletion mutants in complex II (sdh1 4 , sdh2 4 , and sdh4A) showed the shortest CLS among all tested mutants. The mutants that showed decreased CLS included the complex III deletion mutants cor1 $\Delta$, cyt1 $1 \Delta$, qcr7 $\Delta$, qcr8 8 , and rip1 1 , the complex IV mutants cox6 6 , cox7 $\Delta$, and cox9 4 , and the complex $\mathrm{V}$ mutants atp $4 \Delta$, atp $7 \Delta$, and atp17 $\Delta$. The other complex III, IV, and V mutant strains showed CLS that were comparable to that of the wild type (Figs. 1A-1E and Table 1). To independently confirm the CLS of these shortlived KO mutants, we measured CLS using the CFU assay with biological repeats. CFU assay assesses colony formation ability of each cell, and PI assay assesses membrane integrity. Our CLS results showed a consistent pattern of viability; however, the viability pattern of CFU was shorter than PI. This shortened pattern could indicate that cell division mechanism might be damaged faster than membrane integrity during the chronological aging process of yeast (Fig. 1F).

\section{Growth phenotype of ETC deletion mutants}

We measured the $\mathrm{OD}_{600}$ of these mutants during log and early stationary phase growth. The doubling time of the wild type strain was $86 \mathrm{~min}$, and the doubling times of nearly all the KO mutants were comparable to that of wild type, except for atp5 $\Delta$ and atp15 . Deletion of ATP5 and ATP15 prolonged the doubling time $>2$-fold and $>3$-fold compared to that of the wild type strain, respectively (Fig. 2A and Supplementary Table S2A).

We observed differences in the maximum $\mathrm{OD}_{600}$ values of the mutants when we grew them until late stationary phase. Fourteen of the deletion strains showed maximal cell masses that were similar to wild type; however, the maximal cell mass of the remaining 19 strains, sdh $1 \Delta$, sdh2 $\Delta$, sdh $4 \Delta$, cor1 $\Delta$, cyt1 4 , qcr2 4 , qcr7 4 , qcr8 4 , rip1 $1 \Delta$, cox6 4 , cox7 4 , cox9 4 , cox12 $\Delta$, atp1 $\Delta$, atp $4 \Delta$, atp5 $\Delta$, atp7 $\Delta$, atp15 $\Delta$, and atp17 $\Delta$, were half that of wild type (Fig. $2 \mathrm{~B}$ and Supplementary Table S2B). Interestingly, we did not detect a diauxic shift in the growth curves of these strains. In addition, their growth was arrested after the $\mathrm{OD}_{600}$ reached 15 , which was the initial point of the diauxic shift for the wild type strain. Strains with 


\section{A}
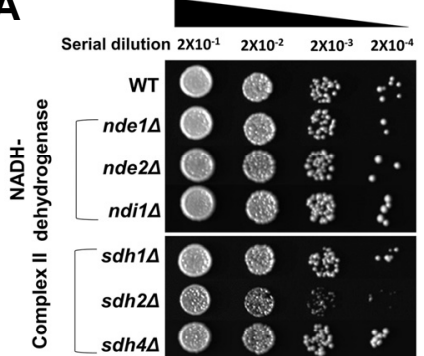

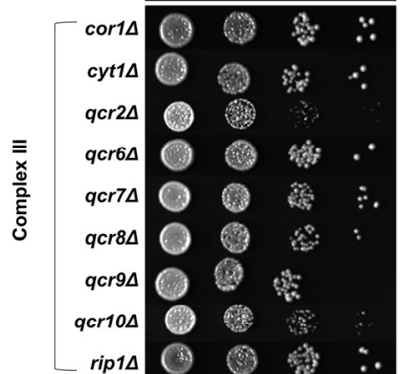

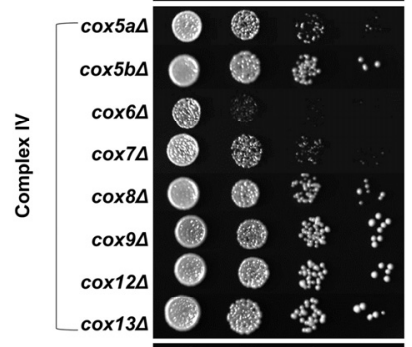

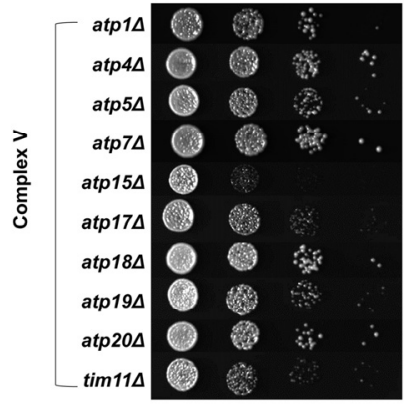

YPD
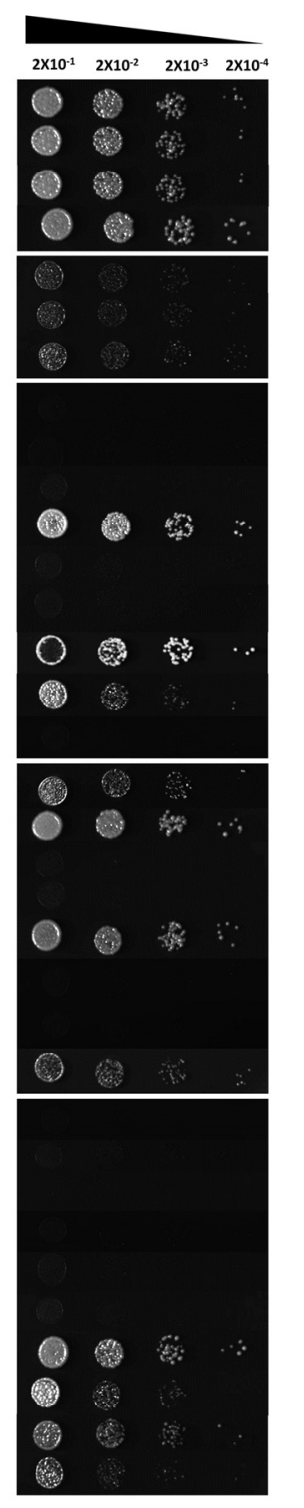

YPG

B

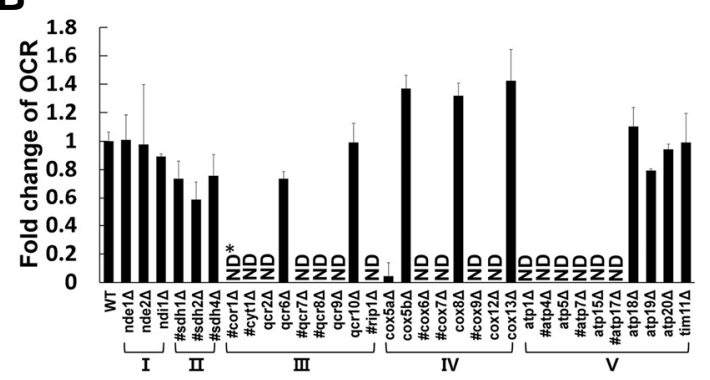

Fig. 3. Fermentative and respiratory growth of ETC single gene deletion mutants. (A) Each mutant strain was spotted on yeast extractpeptone-dextrose (YPD; 2\% glucose) and yeast extract-peptoneglycerol (YPG; 3\% glycerol) agar plates to detect respiratory deficiency. (B) The oxygen consumption rate (OCR) of ETC single gene deletion mutants at day 1 . Error bars indicate standard deviation. " Shortlived strains; ND, Oxygen consumption was not detected.
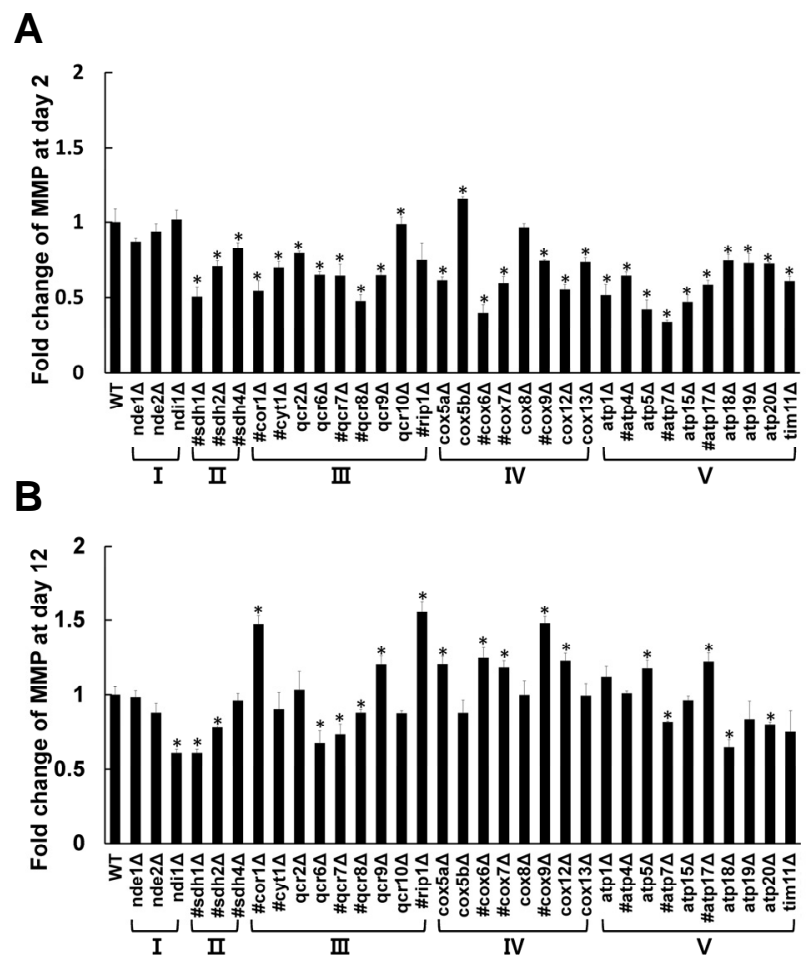

C

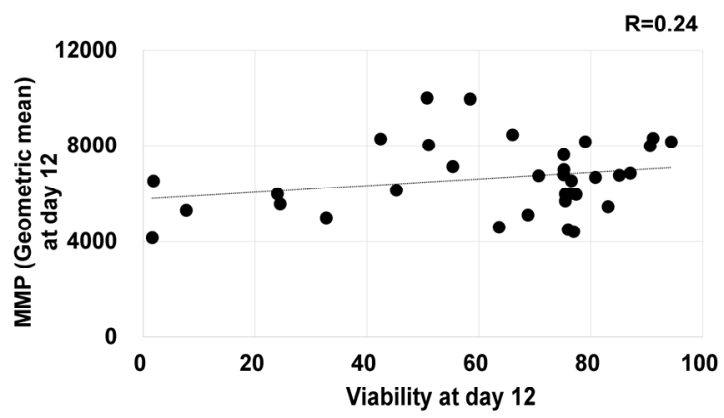

Fig. 4. Mitochondrial membrane potential (MMP) of ETC single gene deletion mutants. (A, B) MMP was measured in ETC single gene deletion mutants at Day $2(A)$ and12 $(B)$, respectively. $(C)$ Correlation between viability and MMP at Day 12. Error bars indicate standard deviation. *Significant difference from wild type $(P<0.05)$; "Shortlived strains.

respiratory deficiency cannot use non-fermentable carbon sources such as ethanol, glycerol, and lactic acid. To assess respiratory deficiency, all KO strains were grown on YP agar plates containing glycerol as a single carbon source. Strains incapable of diauxic shift failed to generate colonies on YP glycerol plates. Nine mutants, sdh1 4 , sdh2 $\Delta$, sdh4 4 , qcr10 4 ,

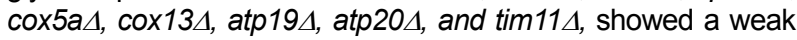
response (Fig. 3A). Furthermore, we measured the oxygen consumption of all KO strains at the post diauxic shift phase, during which the cells mainly grow by respiration. Consistently, strains incapable of diauxic shift could not consume oxygen, and cox5a $\Delta$ had much a lower oxygen consumption rate than the wild type. Exceptionally, qcr9 $\Delta$ could not consume oxygen 
A
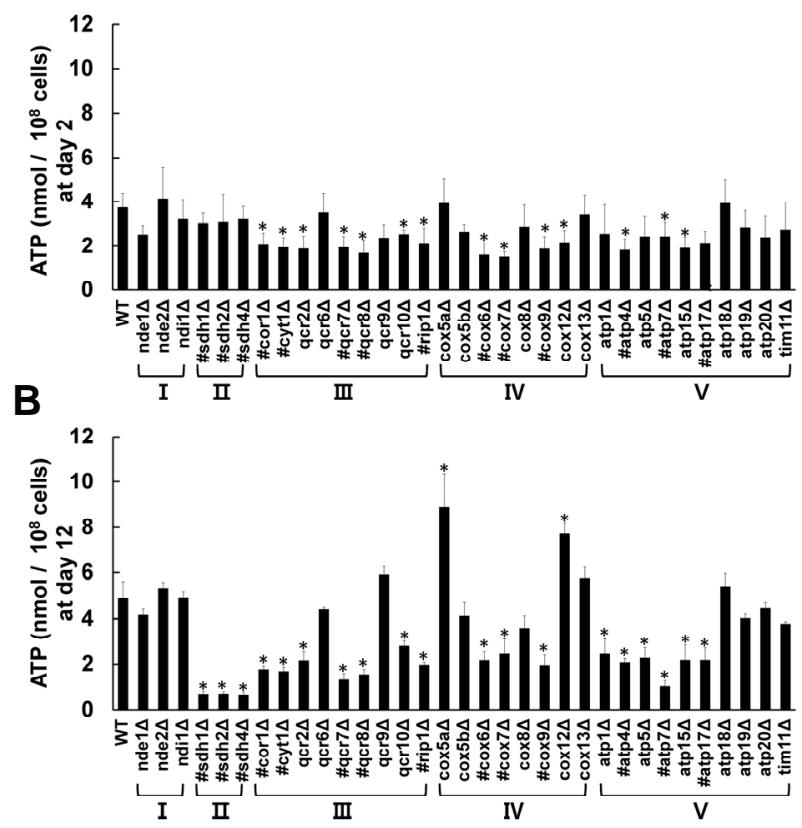

C

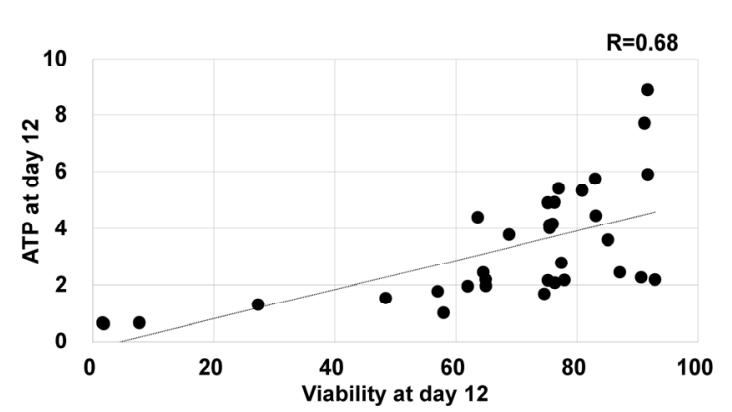

Fig. 5. Intracellular adenosine triphosphate (ATP) levels in ETC single gene deletion mutants. (A, B) Intracellular ATP level was measured in ETC single gene deletion mutants at Day $2(A)$ and12 (B), respectively. (C) Correlation between viability and ATP at Day 12. Error bars indicate standard deviation. *Significant difference from wild type $(P<0.05)$; ${ }^{\#}$ Short-lived strains.

and it showed a weak response with a non-fermentable carbon source (Fig. 3B and Supplementary Table S3). In addition, we analyzed the cell size and complexity of all KO strains using FACS analysis by forward- and side-scatter (FSC and SSC, respectively). Interestingly, the life span of these mutants showed a positive correlation with cell size $(R=0.80)$ but not with cell complexity $(R=0.24)$ (Figs. $2 C$ and $2 D)$.

\section{Effect of a single ETC component deletion on MMP}

The MMP is the major source driving ATP synthesis and other proton-motive-force dependent mitochondrial activities. In order to detect alterations in MMP due to deletion of a single ETC component, we measured the MMP of all KO strains by flow cytometry using $\mathrm{DiOC}_{6}$ fluorescence dye as the indicator during the chronological aging process, particularly at the exponential, post-diauxic, and stationary phases. The MMP of 28 and 19 mutants significantly differ from that of wild type at the post-
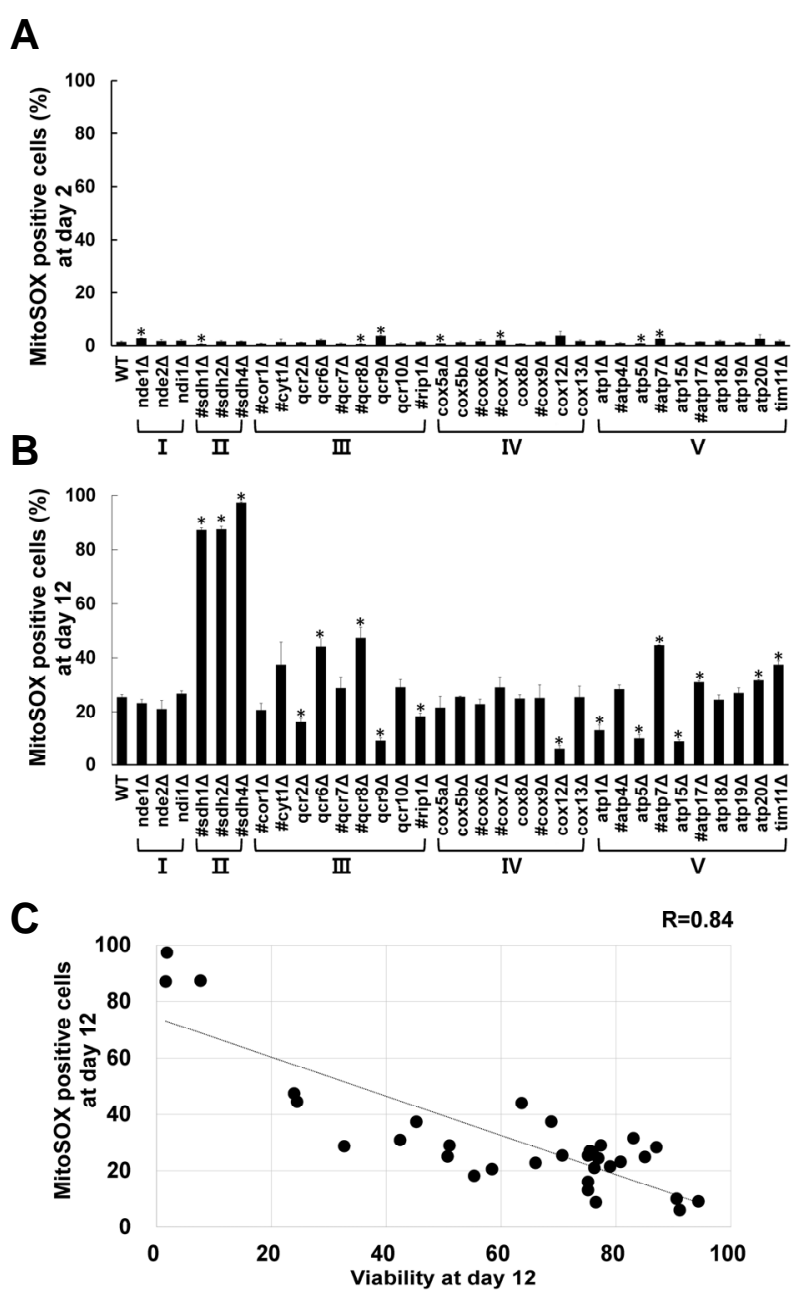

Fig. 6. Mitochondrial superoxide formation in ETC single gene deletion mutants. (A, B) Mitochondrial superoxide production was measured in ETC single gene deletion mutants at Day 2 (A) and12 (B), respectively. (C) Correlation between viability and mitochondrial superoxide formation at Day 12. Error bars indicate standard deviation. *Significant difference from wild type $(P<0.05)$; " Short-lived strains.

diauxic phase and stationary phase, respectively. Although the MMP of these mutants did not significantly differ from that of wild type at the exponential growth, 28 mutants at Day 2 and 19 mutants at Day 12 showed significant alterations in MMP (Figs. $4 A$ and $4 B$, Supplementary Table S4). However, there was no significant correlation between viability and MMP at Day $12(R$ $=0.24$, Fig. 4C).

Intracellular ATP levels in single ETC gene deletion strains ATP is the final product of the ETC and is synthesized by the $\mathrm{F}_{1} \mathrm{~F}_{0}$-ATP synthase (complex $\mathrm{V}$ ) using the proton-motive force in mitochondria. We measured intracellular ATP levels in all KO strains at Days 2 and 12. Fourteen of the strains showed a decrease at Day 2 and nineteen of the strains showed a decrease at Day 12 , while 2 strains ( $\operatorname{cox} 5 \mathrm{a} \Delta$ and $\operatorname{cox} 12 \Delta)$ showed an increase in ATP levels at Day 12 (Figs. 5A and 5B, 

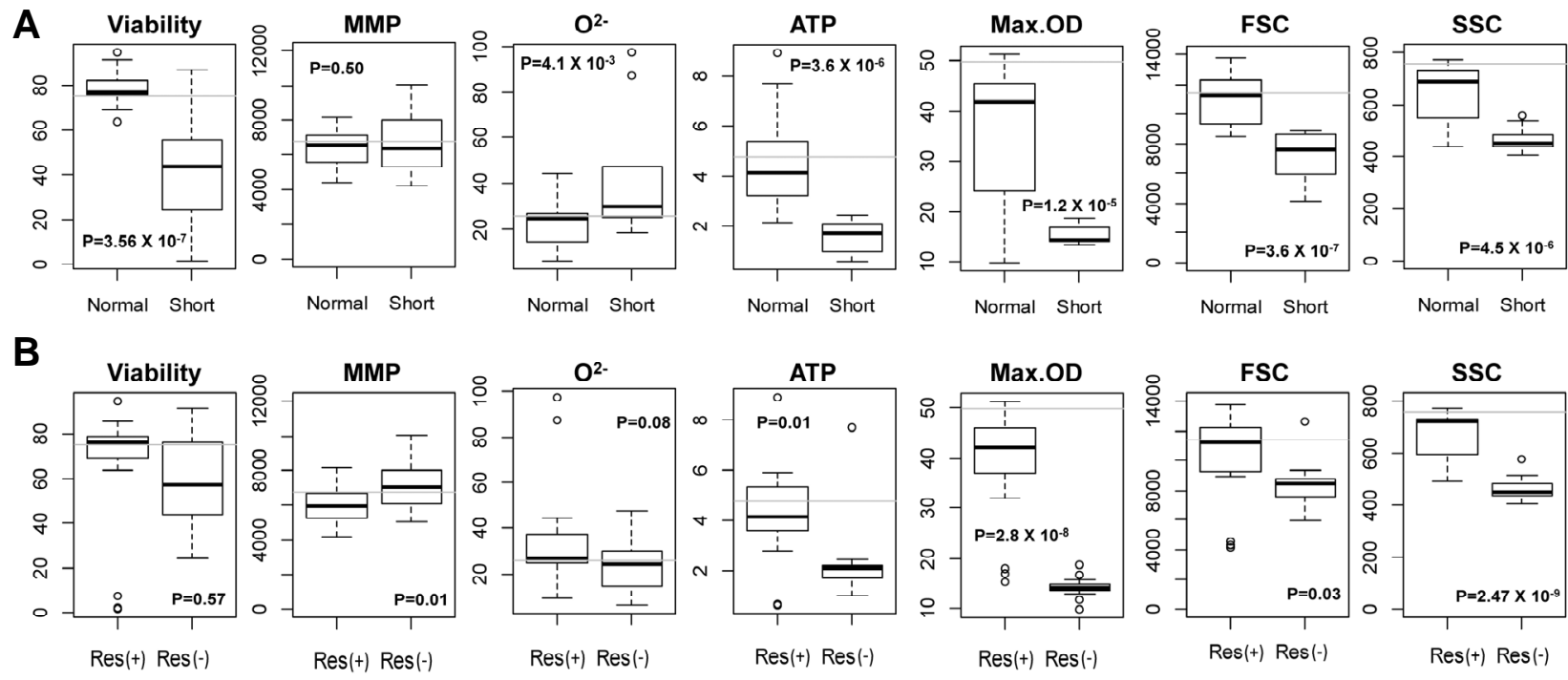

Fig. 7. (A, B) Comparisons between wild type-like mutants and short-lived mutants (A) and respiratory and non-respiratory mutants (B).

Supplementary Table S5). In particular, strains carrying a deletion of a complex II component showed the lowest ATP levels at Day 12, and most respiration-deficient strains had ATP levels lower than that of the wild type strain. ATP levels were clearly positively correlated with viability $(R=0.68$, Fig. $5 C)$.

\section{Generation of mitochondrial superoxide in ETC component deletion mutants}

ROS are byproducts of the ETC respiratory system, and they are mainly produced by complex I and III during electron transport. We measured mitochondrial superoxide levels in all $\mathrm{KO}$ strains during chronological aging at the exponential, postdiauxic shift, and stationary phases using the MitoSOX ${ }^{\mathrm{TM}}$ Red specific indicator. At the exponential and post-diauxic shift phases, mitochondrial superoxide levels were undetectable in all the deletion mutants as well as the wild type strain (Fig. 6A and Supplementary Table S6). However, at day 12, we observed significant alterations in superoxide levels in some of the KO strains. Deletion mutants of complex II-encoding genes, specially $s d h 1 \Delta, s d h 2 \Delta$, and $s d h 4 \Delta$, produced the highest levels of mitochondrial superoxide (Fig. $6 \mathrm{~B}$ and Supplementary Table S6). Furthermore, mitochondrial superoxide generation and viability showed a significant negative correlation $(R=-0.84$, Fig. 6C).

\section{DISCUSSION}

ATP generation is the primary function of mitochondria. However, the mitochondrial ETC also generates deleterious ROS as byproducts. Hence, mitochondria play an important role in producing both essential energy and deleterious molecules in eukaryotic cells. In order to determine the importance of mitochondrial function in the determination of cellular life span, we analyzed the mitochondrial function and CLS of 33 mutants with single gene deletions in ETC components. In our study, 14 ETC deletion strains showed a short CLS, including sdh1 14 ,

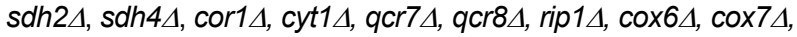
$\operatorname{cox} 9 \Delta$, atp $4 \Delta$, atp7 $\Delta$, and atp17 $\Delta$, whereas the other strains showed normal life span (Fig. 1). Interestingly, all short-lived deletion mutants attained approximately half the mass of the wild type in batch culture (Fig. 2B). Most mutants did not show significant changes in growth rate during exponential phase, except atp5 $\Delta$ and atp15 (Fig. 2A); the growth rates of these 2 strains were prolonged 2- and 3-fold compared to that of wild type, respectively. The genes ATP5 and ATP15 encode oligomycin sensitivity-conferring protein (OSCP) and the epsilon subunit of ATP synthase complex, respectively. Both OSCP (Uh et al., 1990) and the epsilon (Guelin et al., 1993) subunit are part of the ATP synthase complex, which is essential to maintain normal growth rates.

Complex I couples the transfer of electrons from NADH to ubiquinone with the translocation of protons; however, S. cerevisiae lacks complex I. Instead of complex I, reducing equivalents are delivered to the respiratory chain via $3 \mathrm{NADH}$ dehydrogenases encoded by NDE1, NDE2, and NDI1 (JosephHorne et al., 2001). The single gene deletion mutants of NADH dehydrogenase showed wild-type-like CLS, growth, and MMP, ROS, and ATP levels. Previous reports showed that double and triple mutants of NADH dehydrogenase components exhibited impaired respiratory growth, whereas the single deletion mutants did not (Gomes et al., 2013). Therefore, single gene deletions of NADH dehydrogenase components are insufficient to impair yeast growth.

Succinate dehydrogenase (SDH) plays a central role in the ETC and TCA cycle as an oxidase that converts succinate to fumarate and ubiquinone to ubiquinol. Mutants with a disruption of $S D H 1, S D H 2$, or $S D H 4$ showed a shortened life span and reduced mitochondrial efficiency due to lower ATP levels and higher mitochondrial superoxide levels, while maintaining MMP levels.

Complex III (cytochrome bc1 complex) is well-characterized as a major respiratory component in mammals and it catalyzes electron transport from ubiquinol to cytochrome c. We showed that several complex III respiratory deficient mutants, including cyt1 4 , cor1 4 , qcr2 4 , qcr7 $\Delta$ and qcr8 4 , had shortened life span.

COX4, COX6, COX7, and COX9 encode key components required for cytochrome $\mathrm{C}$ oxidase activity. In contrast, other genes in complex IV are not essential for this activity (Aggeler 
Table 2. Phenotype summary of the studied ETC KO strains

\begin{tabular}{|c|c|c|c|c|c|c|c|}
\hline Complex & Strains & CLS & $\begin{array}{l}\text { Maximum } \\
\text { cell mass }\end{array}$ & OCR & YPG & ROS & ATP \\
\hline \multirow{3}{*}{$\begin{array}{c}\mathrm{NADH} \\
\text { dehydrogenase }\end{array}$} & nde1 14 & $\leftrightarrow$ & $\leftrightarrow$ & $\leftrightarrow$ & $\leftrightarrow$ & $\leftrightarrow$ & $\leftrightarrow$ \\
\hline & nde2 $\Delta$ & $\leftrightarrow$ & $\leftrightarrow$ & $\leftrightarrow$ & $\leftrightarrow$ & $\leftrightarrow$ & $\leftrightarrow$ \\
\hline & ndi14 & $\leftrightarrow$ & $\leftrightarrow$ & $\leftrightarrow$ & $\leftrightarrow$ & $\leftrightarrow$ & $\leftrightarrow$ \\
\hline \multirow[t]{3}{*}{ II } & $\operatorname{sdh} 1 \Delta$ & $\nabla$ & $\nabla$ & $\leftrightarrow$ & $\nabla \nabla$ & $\triangle$ & $\nabla$ \\
\hline & $s d h 2 \Delta$ & $\nabla$ & $\nabla$ & $\leftrightarrow$ & $\nabla \nabla$ & $\triangle$ & $\nabla$ \\
\hline & $s d h 4 \Delta$ & $\nabla$ & $\nabla$ & $\leftrightarrow$ & $\nabla \nabla$ & $\triangle$ & $\nabla$ \\
\hline \multirow[t]{9}{*}{ III } & cor1 $\triangle$ & $\nabla$ & $\nabla$ & $\nabla \nabla \nabla$ & $\nabla \nabla \nabla$ & $\leftrightarrow$ & $\nabla$ \\
\hline & cyt14 & $\nabla$ & $\nabla$ & $\nabla \nabla \nabla$ & $\nabla \nabla \nabla$ & $\leftrightarrow$ & $\nabla$ \\
\hline & $q c r 2 \Delta$ & $\leftrightarrow$ & $\nabla$ & $\nabla \nabla \nabla$ & $\nabla \nabla \nabla$ & $\nabla$ & $\nabla$ \\
\hline & qcr6 6 & $\leftrightarrow$ & $\leftrightarrow$ & $\nabla$ & $\leftrightarrow$ & $\triangle$ & $\leftrightarrow$ \\
\hline & qcr7 4 & $\nabla$ & $\nabla$ & $\nabla \nabla \nabla$ & $\nabla \nabla \nabla$ & $\leftrightarrow$ & $\nabla$ \\
\hline & qcr84 & $\nabla$ & $\nabla$ & $\nabla \nabla \nabla$ & $\nabla \nabla \nabla$ & $\triangle$ & $\nabla$ \\
\hline & qcr9s & $\leftrightarrow$ & $\leftrightarrow$ & $\nabla \nabla \nabla$ & $\leftrightarrow$ & $\nabla$ & $\leftrightarrow$ \\
\hline & qcr10 & $\leftrightarrow$ & $\leftrightarrow$ & $\leftrightarrow$ & $\nabla$ & $\leftrightarrow$ & $\nabla$ \\
\hline & rip14 & $\nabla$ & $\nabla$ & $\nabla \nabla \nabla$ & $\nabla \nabla \nabla$ & $\nabla$ & $\nabla$ \\
\hline \multirow[t]{8}{*}{ IV } & $\operatorname{cox} 5 a \Delta$ & $\leftrightarrow$ & $\nabla$ & $\nabla \nabla$ & $\nabla$ & $\leftrightarrow$ & $\triangle$ \\
\hline & $c o x 5 b \Delta$ & $\leftrightarrow$ & $\leftrightarrow$ & $\triangle$ & $\leftrightarrow$ & $\leftrightarrow$ & $\leftrightarrow$ \\
\hline & $\operatorname{cox} 6 \Delta$ & $\nabla$ & $\nabla$ & $\nabla \nabla \nabla$ & $\nabla \nabla \nabla$ & $\leftrightarrow$ & $\nabla$ \\
\hline & $\cos 7 \Delta$ & $\nabla$ & $\nabla$ & $\nabla \nabla \nabla$ & $\nabla \nabla \nabla$ & $\leftrightarrow$ & $\nabla$ \\
\hline & $\operatorname{cox} 8 \Delta$ & $\leftrightarrow$ & $\leftrightarrow$ & $\leftrightarrow$ & $\leftrightarrow$ & $\leftrightarrow$ & $\leftrightarrow$ \\
\hline & $\operatorname{cox} 9 \Delta$ & $\leftrightarrow$ & $\nabla$ & $\nabla \nabla \nabla$ & $\nabla \nabla \nabla$ & $\leftrightarrow$ & $\nabla$ \\
\hline & $\operatorname{cox} 12 \Delta$ & $\leftrightarrow$ & $\nabla$ & $\nabla \nabla \nabla$ & $\nabla \nabla \nabla$ & $\nabla$ & $\triangle$ \\
\hline & $\operatorname{cox} 13 \Delta$ & $\leftrightarrow$ & $\leftrightarrow$ & $\leftrightarrow$ & $\nabla$ & $\leftrightarrow$ & $\leftrightarrow$ \\
\hline \multirow[t]{10}{*}{ V } & atp1ム & $\leftrightarrow$ & $\nabla$ & $\nabla \nabla \nabla$ & $\nabla \nabla \nabla$ & $\nabla$ & $\nabla$ \\
\hline & atp4 $\Delta$ & $\nabla$ & $\nabla$ & $\nabla \nabla \nabla$ & $\nabla \nabla \nabla$ & $\leftrightarrow$ & $\nabla$ \\
\hline & $\operatorname{atp} 5 \Delta$ & $\leftrightarrow$ & $\nabla$ & $\nabla \nabla \nabla$ & $\nabla \nabla \nabla$ & $\nabla$ & $\nabla$ \\
\hline & atp7 4 & $\nabla$ & $\nabla$ & $\nabla \nabla \nabla$ & $\nabla \nabla \nabla$ & $\triangle$ & $\nabla$ \\
\hline & atp15 & $\leftrightarrow$ & $\nabla$ & $\nabla \nabla \nabla$ & $\nabla \nabla \nabla$ & $\nabla$ & $\nabla$ \\
\hline & atp17ム & $\nabla$ & $\nabla$ & $\nabla \nabla \nabla$ & $\nabla \nabla \nabla$ & $\triangle$ & $\nabla$ \\
\hline & atp184 & $\leftrightarrow$ & $\leftrightarrow$ & $\leftrightarrow$ & $\leftrightarrow$ & $\leftrightarrow$ & $\leftrightarrow$ \\
\hline & atp19A & $\leftrightarrow$ & $\leftrightarrow$ & $\nabla$ & $\nabla$ & $\leftrightarrow$ & $\leftrightarrow$ \\
\hline & atp20 $\Delta$ & $\leftrightarrow$ & $\leftrightarrow$ & $\leftrightarrow$ & $\nabla$ & $\triangle$ & $\leftrightarrow$ \\
\hline & $\operatorname{tim} 11 \Delta$ & $\leftrightarrow$ & $\leftrightarrow$ & $\leftrightarrow$ & $\nabla$ & $\triangle$ & $\leftrightarrow$ \\
\hline
\end{tabular}

Symbols indicate a significant $(\mathrm{P}$ value $<0.05)$ increase $(\triangle)$, decrease $(\boldsymbol{\nabla})$, or no change $(\leftrightarrow)$.

The number of symbols indicate the extent.

Significant difference for maximum cell mass counts both fold-change $>|2|$ and $P$ value $<0.05$.

and Capaldi, 1990). Interestingly, single deletion mutants of COX6, COX7, and COX9 showed a shortened CLS in our study. We did not test COX4 because this deletion strain was not available in the deletion library. Our results suggest that cytochrome $\mathrm{C}$ oxidase activity is essential for maintaining a normal life span. The COX5p isoforms encoded by either COX5a or COX5b are components of cytochrome c oxidase subunit V. COX5a is primarily expressed under aerobic conditions, whereas $C O X 5 b$ is primarily expressed under anaerobic conditions. Cox5a deletion mutant had only $6 \%$ of the cytochrome $\mathrm{c}$ oxidase activity and respiration rate of the wild type strain, whereas cox $5 b \Delta$ was similar to wild type (Trueblood and
Poyton, 1987). Consistently, our results also showed that the respiratory growth of $\operatorname{cox} 5 \mathrm{a} \Delta$ was decreased during chronological aging, whereas that of cox $5 b \Delta$ was not. However, despite the reduced cytochrome c oxidase activity of the cox $5 a \Delta$ strain, its CLS was similar to that of the wild type strain.

Among the complex $\mathrm{V}$ mutants, the single deletion mutants of ATP4, ATP7, and ATP17, encoding the b, d, and f subunits of ATP synthase, respectively, showed a shortened CLS. Both subunit $b$ and $d$ are components of the stator stalk of ATP synthase. Therefore, the absence of these genes could lead to structural instability of the $F_{1} F_{0}$ complex (Norais et al., 1991; Velours et al., 1989). 
MMP is a pre-requisite for the generation of ATP by the ETC in the mitochondrial inner membrane. We detected no correlation between viability and MMP levels; however, viability was positively correlated with ATP levels and negatively correlated with mitochondrial superoxide levels (Figs. 4B, 5B, and 6B). Null mutations of ETC components impair the flow of protons in the mitochondrial ETC system. Our results suggest that an impaired mitochondrial ETC system affects longevity by decreasing mitochondrial efficiency by uncoupling MMP from ATP production, which increases the generation of ROS by electron leakage. The decreased ATP might cause consequences of the smaller cell size and approximately half cell mass in the shortlived mutants. In addition, several organic acids could be accumulated in the culture medium during the chronological aging process, and they cause $\mathrm{pH}$ change. Although we did not test these factors, they may also limit CLS of these mutants.

At the stationary phase, the short-lived strains had lower cell mass $\left(P=1.2 \times 10^{-5}\right)$, cell size $\left(P=3.6 \times 10^{-7}\right)$, cell complexity $\left(P=4.5 \times 10^{-8}\right)$, and ATP production $\left(P=3.6 \times 10^{-8}\right)$, but higher mitochondrial superoxide levels $\left(P=4.1 \times 10^{-3}\right)$ than the mutants with normal life span. However, the MMP levels did not differ significantly between these 2 groups (Fig. 7A). These results suggest the presence of efficient mitochondria that produce more ATP than ROS when the MMP is sufficient. We also analyzed the effect of respiration on viability. We separated the 33 mutants into 2 groups, respiratory or non-respiratory. We did not observe a difference in the viability and superoxide production between them. The non-respiratory mutants showed higher MMP levels $(P=0.01)$, but lower ATP levels $(P=0.01)$, than the respiratory mutants. As expected, respiration deficiency due to mutation decreased cell mass $\left(P=1.8 \times 10^{-8}\right)$, cell size $(P=$ $0.03)$, and cell complexity $\left(P=2.47 \times 10^{-9}\right)$ (Fig. $\left.7 \mathrm{~B}\right)$. In conclusion, these results suggest that mitochondrial efficiency, but not respiration, is the prerequisite to a longer life span.

Note: Supplementary information is available on the Molecules and Cells website (www.molcells.org).

\section{ACKNOWLEDGMENTS}

This work was supported by the "Cooperative Research Program for Agriculture Science \& Technology Development (Project No. PJ01053302)" of the Rural Development Administration, and the Bio \& Medical Technology Development Program of the National Research Foundation (NRF) funded by the Ministry of Science, ICT \& Future Planning (NRF-20110030137), Republic of Korea. The corresponding author thanks Sang-Mi Park for measuring $\mathrm{OD}_{600}$ values in the culture.

\section{REFERENCES}

Aggeler, R., and Capaldi, R.A. (1990). Yeast cytochrome c oxidase subunit VII is essential for assembly of an active enzyme. Cloning, sequencing, and characterization of the nuclearencoded gene. J. Biol. Chem. 265, 16389-16393.

Bratic, A., and Larsson, N.G. (2013). The role of mitochondria in aging. J. Clin. Invest. 123, 951-957.

Breitenbach, M., Laun, P., Dickinson, J.R., Klocker, A., Rinnerthaler, M., Dawes, I.W., Aung-Htut, M.T., Breitenbach-Koller, L., Caballero, A., Nystrom, T., et al. (2012). The role of mitochondria in the aging processes of yeast. Subcell. Biochem. 57, 55-78.

Choi, J.S., and Lee, C.K. (2013). Maintenance of cellular ATP level by caloric restriction correlates chronological survival of budding yeast. Biochem. Biophys. Res. Commun. 439, 126-131.

Choi, J.S., Choi, K.M., and Lee, C.K. (2011). Caloric restriction improves efficiency and capacity of the mitochondrial electron transport chain in Saccharomyces cerevisiae. Biochem. Biophys. Res. Commun. 409, 308-314.
Choi, K.M., Kwon, Y.Y., and Lee, C.K. (2013a). Characterization of global gene expression during assurance of lifespan extension by caloric restriction in budding yeast. Exp. Gerontol. 48, 14551468.

Choi, K.M., Lee, H.L., Kwon, Y.Y., Kang, M.S., Lee, S.K., and Lee, C.K. (2013b). Enhancement of mitochondrial function correlates with the extension of lifespan by caloric restriction and caloric restriction mimetics in yeast. Biochem. Biophys. Res. Commun. 441, 236-242.

Choi, K.M., Kwon, Y.Y., and Lee, C.K. (2015). Disruption of Snf3/Rgt2 glucose sensors decreases lifespan and caloric restriction effectiveness through Mth1/Std1 by adjusting mitochondrial efficiency in yeast. FEBS Lett. 589, 349-357.

de Grey, A.D. (2005). Reactive oxygen species production in the mitochondrial matrix: implications for the mechanism of mitochondrial mutation accumulation. Rejuvenation Res. 8, 13-17.

Demir, A.B., and Koc, A. (2010). Assessment of chronological lifespan dependent molecular damages in yeast lacking mitochondrial antioxidant genes. Biochem. Biophys. Res. Commun. 400, 106-110.

Duttaroy, A., Paul, A., Kundu, M., and Belton, A. (2003). A Sod2 null mutation confers severely reduced adult life span in Drosophila. Genetics 165, 2295-2299.

Gerschman, R., Gilbert, D.L., Nye, S.W., Dwyer, P., and Fenn, W.O. (1954). Oxygen poisoning and x-irradiation: a mechanism in common. Science 119, 623-626.

Gomes, F., Tahara, E.B., Busso, C., Kowaltowski, A.J., and Barros, M.H. (2013). nde1 deletion improves mitochondrial DNA maintenance in Saccharomyces cerevisiae coenzyme Q mutants. Biochem. J. 449, 595-603.

Gralla, E.B., and Kosman, D.J. (1992). Molecular genetics of superoxide dismutases in yeasts and related fungi. Adv. Genet. 30, 251-319.

Guelin, E., Chevallier, J., Rigoulet, M., Guerin, B., and Velours, J. (1993). ATP synthase of yeast mitochondria. Isolation and disruption of the ATP epsilon gene. J. Biol. Chem. 268, 161-167.

Hacioglu, E., Demir, A.B., and Koc, A. (2012). Identification of respiratory chain gene mutations that shorten replicative life span in yeast. Exp. Gerontol. 47, 149-153.

Harman, D. (1956). Aging: a theory based on free radical and radiation chemistry. J. Gerontol. 11, 298-300.

Harman, D. (1972). The biologic clock: the mitochondria? J. Am. Geriatr. Soc. 20, 145-147.

Joseph-Horne, T., Hollomon, D.W., and Wood, P.M. (2001). Fungal respiration: a fusion of standard and alternative components. Biochim. Biophys. Acta 1504, 179-195.

Kaeberlein, M., Kirkland, K.T., Fields, S., and Kennedy, B.K. (2005) Genes determining yeast replicative life span in a long-lived genetic background. Mech. Ageing Dev. 126, 491-504.

Lee, Y.L., and Lee, C.K. (2008). Transcriptional response according to strength of calorie restriction in Saccharomyces cerevisiae. Mol. Cells 26, 299-307.

Li, Y., Huang, T.T., Carlson, E.J., Melov, S., Ursell, P.C., Olson, J.L., Noble, L.J., Yoshimura, M.P., Berger, C., Chan, P.H., et al. (1995) Dilated cardiomyopathy and neonatal lethality in mutant mice lacking manganese superoxide dismutase. Nat. Genet. 11, 376381.

McCord, J.M., and Fridovich, I. (1969). Superoxide dismutase. An enzymic function for erythrocuprein (hemocuprein). J. Biol. Chem. 244, 6049-6055.

Mitchell, P. (1961) Coupling of phosphorylation to electron and hydrogen transfer by a chemi-osmotic type of mechanism. Nature 191, 144-148.

Muller, F.L., Liu, Y., and Van Remmen, H. (2004). Complex III releases superoxide to both sides of the inner mitochondrial membrane. J. Biol. Chem. 279, 49064-49073.

Norais, N., Prome, D., and Velours, J. (1991). ATP synthase of yeast mitochondria. Characterization of subunit $d$ and sequence analysis of the structural gene ATP7. J. Biol. Chem. 266, 1654116549.

Passos, J.F., von Zglinicki, T., and Saretzki, G. (2006). Mitochondrial dysfunction and cell senescence: cause or consequence? Rejuvenation Res. 9, 64-68.

Scialo, F., Mallikarjun, V., Stefanatos, R., and Sanz, A. (2013). Regulation of lifespan by the mitochondrial electron transport chain: reactive oxygen species-dependent and reactive oxygen 
species-independent mechanisms. Antioxid. Redox Signal. 19, 1953-1969.

Stehr-Green, P.A., Cochi, S.L., Preblud, S.R., and Orenstein, W.A (1990). Evidence against increasing rubella seronegativity among adolescent girls. Am. J. Public Health $80,88$.

Trueblood, C.E., and Poyton, R.O. (1987). Differential effectiveness of yeast cytochrome $c$ oxidase subunit genes results from differences in expression not function. Mol. Cell. Biol. 7, 35203526.

Uh, M., Jones, D., and Mueller, D.M. (1990). The gene coding for the yeast oligomycin sensitivity-conferring protein. J. Biol. Chem.
265, 19047-19052.

Van Raamsdonk, J.M., and Hekimi, S. (2009). Deletion of the mitochondrial superoxide dismutase sod-2 extends lifespan in Caenorhabditis elegans. PLoS Genet. 5, e1000361.

Veatch, J.R., McMurray, M.A., Nelson, Z.W., and Gottschling, D.E. (2009). Mitochondrial dysfunction leads to nuclear genome instability via an iron-sulfur cluster defect. Cell 137, 1247-1258.

Velours, J., Arselin, G., Paul, M.F., Galante, M., Durrens, P., Aigle, M., and Guerin, B. (1989). The yeast ATP synthase subunit 4: structure and function. Biochimie 71, 903-915. 